\title{
Ueber nutzbare australische Bäume
}

berichtet Herr Carl Wilhelmi (in den Sitzungsberichten der Naturwiss. Gesellsch. I s i s in Dresden, Jahrg. 1871, Mai, Juni, Juli, S. 100-104). Am meisten sind es die Myrtaceen, welche die Aufmerksamkeit auf sich lenken und darunter wieder die Eucalypten, welche den Hauptcharacter der australischen Landschaft ausmachen und wegen ihrer Masse, so wie colossalen Grösse und Dauerhaftigkeit ihres Holzes bemerkenswerth sind. Unter diesen Eucalypten sind hauptsächlich folgende hervorzubeben:

Eucalyptus globulus Labil., Blue Gum, welcher in grossen Massen in den Küstenstrichen von Victoria und der Insel Tasmania anzutreffen ist, verdient wegen seines ausgezeichneten Holzes, schnellen Wachstbums und enormer Grösse den Vorrang vor allen Anderen. Das Holz wird in den Colonien zum Schiffbau und wegen seiner Dauerhaftigkeit zu Eisenbahnschwellen, Brückenbauten und Wasserwerken aller Art und zu allem nur Denklichen benutzt, wo lange Daner nothwendig ist, da es dem Holze unserer Eiche gleichkommt und an Dicke des Stammes nur dem indischen Affenbrodbaume (Adans onia digitata) nachsteht. Die schönen, geraden Stämme erreichen eine Höhe von 70-86 Meter (250 - 300 Fuss), bei einer Dicke von 7 Meter (25 Fuss), während die Aeste gewöhnlich erst in einer Höhe von $34 \mathrm{Me}-$ ter (120 Fuss anfangen). Unser bei der Bourke- und Wills'schen Expedition umgekommener Landemann Dr. L. Becker giebt das Maass eines von ihm in Tasmania getundenen E. globulus, wie folgt:

Umfang des Stammes nahe dem Grunde 26 Met. (90 Fuss),

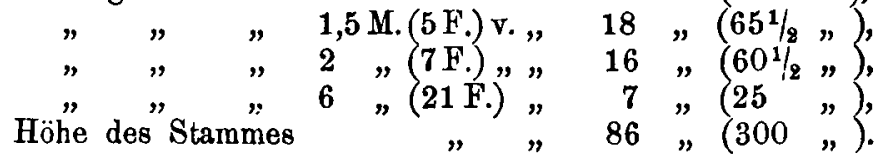

Eucalyptus rostrata Cav., Red Gum, ein Baum, welcher eine gleiche Höhe erreicht, liefert nach $\mathrm{E}$. globulus eines der nützlichsten Hölzer Australiens und ist fast über alle Colonien in Menge verbreitet. Dies Holz ist spröde, aber ausgezeichnet für Wasserbauten, so wie Eisenbahnschwellen und wird, da es eine herrliche Politur annimmt, auch zu Hausgeräthen verarbeitet.

Eucalyptus fabrorum, Sringy Bark; sein Holz ist das in den Colonien am meisten gebrauchte, weil es 
ungemein leicht spaltet und werden die feinsten Dachschindeln, so wie dicke Bretter und Pfosten für Einzäunungen davon gespalten, während die Rinde gewöhnlich zum Decken der Häuser benutzt wird, ja sogar zur Fertigung eines groben Papieres dienen kann.

Eucalyptus acervula Sieb., White Gum, wird von dem in Australien Reisenden mit Freuden begrüsst, da es stets das Vorhandensein von Flüssen, Bächen oder Wasserlöchern anzeigt und in ansehnlicher Grösse an denselben wächst. Das Holz ist dem von E. rostrata Cav. ähnlich, nur von blasser Farbe. Von der sich leicht schälenden, zolldicken Rinde dieses Baumes verfertigen die Eingebornen ihre Schilder, Canoos, sowie Schutzdächer während der Regenzeit. Die Häuser der im Busch lebenden Europäer werden ebenfalls mit dieser Rinde gedeckt, ja selbst ganze Häuser davon gebaut, welche 10-12 Jahre stehen, ehe sie baufällig werden. Die Aussenseite der abgeschälten Rinde wird mit heisser Asche oder Kohlen bestreut, damit sich dieselbe gerade zieht und sich nicht wirft (nicht rollt).

Eucalyptus resinifera Sm., Ironbark; das Holz ist sehr danerhaft, aber wegen seiner Härte schlecht zu bearbeiten; gewöhnlich werden Wagenrïder daraus gemacht. Dieser Baum wächst in steinigem Gebirgsboden und ist hauptsächlich auf den Goldfeldern stark vertreten. Die Rinde ist fast schwarz und tief gefurcht. Er erreicht eine Höhe von 43 Meter (150 Fuss) bei 0,7 M. (21/2 F.) Durchmesser.

Eucalyptus amygdalina Labil., Pepperminth oder Oil Gum genannt, ist seiner colossalen Höhe, scines Holzes und seiner Blätter wegen interessant. In einem Gebirgsthale nahe Lillydale in Victoria stehen mehre Bäume beisammen, welche die colossale Höhe von 120 Meter (420 Fuss) haben. Trotz dieser Höhe ist der Stanm 0,861,14 Meter (3-4 Fuss) von der Erde nur 1,4-2 Meter (5-6 Fuss) im Durchmesser und so schlank wie ein Mast.

Die Blätter aller Eucalypten sind reichhaltig an ätherischem Oel, welches dem Cajeputöle von Indien gleich kommt und wegen seiner campherartigen Natur in der Medicin, sowie in der Parfümerie benutzt wird. Hauptsächlich ist es aber dieser Eucalyptus, welcher das meiste $\mathrm{Oel}$ liefert und zwar 4 Pfund von 100 Pfund Blättern, während Melaleuca linarifolia Sm. die nächstgrösşte Quantität und zwar $1 \frac{3}{4}$ Pfund Oel von 100 Pfund Blättern liefert.

In einem kleinen Städtchen unweit Melbourne sind diese Eucalyptenblätter, da der ganze australische Wald 
fast nur aus Eucalypten besteht, zur Gasbereitung und zwar mit Erfolg benutzt worden.

Unter den Acacien sind hauptsächlich hervorzuheben: Acacia Melanoxylon R. Br., Blackwood, ein herrliches, dauerhaftes, leicht zu spaltendes und gute Politur annehmendes $\mathrm{Holz}$, welches nicht springt und sich weniger wirft, als irgend ein Holz in Australien und mit unserem Wallnussholze verglichen werden kann. In den Colonien wird es zu Eisenbahnwagen, Schiffbrïcken n. a. mögl. Möbeln verarbeitet und wächst hauptsächlich in feuchten Wäldern zu einer Höhe von 35 Meter (120 Fuss) mit einem geraden Stamme von $0,56-0,86$ M. (2-3 F.) Durchmesser.

Acacia ctenophylla, wächst am Murray-Flusse, und steht ihres herrlichen Holzes wegen der Vor. wenig nach.

Cedrela a ustralis, a ustralische Ceder, die in Ost-Australien vorkommt, liefert ein, die schönste Politur annehmendes Holz, ist leicht $\mathrm{za}$ bearbeiten und wird zu allen mögl. Möbeln benutzt. Fast alle die bis jetzt angefuihrten Nutzbölzer werden nicht allein in den Colonien verarbeitet, sondern sind schon Ausfuhrartikel.

Fagus Cunninghami, Native Beech, kommt nur in feuchten Gebirgsthälern von Victoria und Tasmania vor; erreicht ein Höhe von 22 bis 28 Meter $(80 \ldots 100$ Fuss) mit einem Durchmesser des Stammes von 0,56 M. (2 Fuss). Das Holz nimmt eine gute Politur an, ist aber nicht so dauerhaft, wie das unserer europäischen Buche.

Toryphora Sassafras, Sassafrastree, erreicht eine Höhe von 14-17 Meter $(50-60 \mathrm{~F}$ ), während der Durchmesser des Stammes 0,4 bis $0,6 \mathrm{M}$. (1/2 - 2 F.) beträgt. Dieser Baum wächst nur in den feuchten Thälern von $\mathrm{Victoria}$ und Tasmania und ist wegen seiner bitteren $R$ inde, welche jetzt schon in grossen Massen ausser Landes geht, werthvoll in der Medicin geworden. Das Holz, wenn polirt, sieht unserm Nussbaumholz ähnlich.

Panax dendroides, Mountain Ash, nur in Gebirgen vorkommend, erreicht eine Höhe von $8,5-11,5 \mathrm{M}$. (30 bis 40 F.) bei einem Durchmesser von 20 Centim. (9 Zoll). Ias leichte, zähe Holz besitzt die Güte unseres Eschenholzes.

Melaleuca squarrosa $\mathrm{Smith}$ ist in fenchten Thälern als ein Baum von $22-28$ M. $\left(80-100^{\prime}\right)$ Höhe und mit einem Stamme von $0,4-0,6$ M. (1/2-2') Durchmesser anzutreffen. Das blassrothe Holz ist fein und dauerhaft und 
kann za Möbeln und Drechslerarbeiten sehr gut verwendet werden.

Acmena floribunda Dc., Myrtle Tree of Sealers Cove, wird in brauchbaren Exemplaren in feuchten Gebirgsthälern von Gipsland bis zu einer Höhe von $14 \mathrm{M}$. $\left(50^{\prime}\right)$, bei einem Durchmesser von 0,5 M. $\left(1^{1} / 2^{\prime}\right)$ gefunden. Sein zähes, hartes Holz wird hauptsächlich zu Maschineriearbeiten benutzt.

Das Holz von Notelaea ligustrina Vent., Pomaderris apetala Labil. und Lomatia Fraseri R. Br. hat dieselben Eigenschaften, wie das von Acmene, und denselben Standort, nur werden die drei genannten Bäume stärker und ihr Holz nirnmt eine schöne Politur an.

Dasselbe gilt von der $6 \mathrm{M}$. $\left(20^{\prime}\right)$ hohen und $0,28 \mathrm{M}$. dicken Banksia integrifolia und der $8,6 \mathrm{M}$. (30) hohen 0,6 M. (2') dicken Banksia australis, welche ein sehr schön gezeichnetes $\mathrm{Holz}$ besitzen.

Callitris (Frenela) Preissii und C. cupressiformis Sweet., Murray Pine werden hauptsächlich in grösseren Wäldern an den sandigen Ufern des MurrayFlusses angetroffen und erreichon daselbst eine Höh von $13-14 \mathrm{M}$. $\left(40-50^{\prime}\right)$. Das is das einzige Holz, welches in Folge seiner Leichtigkeit zu Flössen benutzt worden kann. Auch werden von den geraden 0,28 M. (1) dicken Stämmen gewöhnl. die Häuser der Ansiedler gebaut, welche durch die horizontal auf einander gefügten Stämme ein recht sauberes Ansehen haben. Um diese Häuser dicht zu machen, werden die Spalten zwischen den unbehauenen Stämmen mit Moos verstopft und dann mit Lehm verklebt.

Casuarina quadrivalvis Lab., Sheioak und C. leptoclada, Heoak, sind in grossen Wäldern anzutreffen, welche wegen ihrer dunkeln schachtelhalmartigen Blattbildung merkwürdig sind uad der Landschaft einon fast trauernden Charakter verleihen. Die Bäume erreichen eine Höbe von $5-8 \mathrm{M}$. $\left(20-30^{\prime}\right)$ und eine Dicke vón $0,28 \mathrm{M}$. C. quadrivalvis wächst meistens in sandigen, unfruchtbaren Gegenden, und in trocknen Jahreszeiten oder grasarmen Gegenden dienen die säuerlich schmeckenden Blätter oft aushülfsweise als Viehfutter; C. l eptocla da ist meistens nur auf feuchtem Boden anzutreffen und wird auch von den Ansiedlern $\mathrm{Swamp-Oak}$ genannt. Letztere hat starke aufrechtstehende Blätter, während die der ersteren hängen.

Callistemon salignum D. C., S tonewood, kommt unserem Buxbaumholze ziemlich gleich und kann, wie dieses, 
zup Xylographie verwendet werden; es wird nur 3,5-4 M. $\left(12-15^{\prime}\right)$ hoch.

Bursaria spinosa Cav., Boxwood, welche $5-8 \mathrm{M}$. $\left(20-30^{\circ}\right)$ erreicht, dient zu gleichen Zwecken.

Aster argophyllus Labil., von den Ansiedlern wegen des starken Moschusgeruches der Blätter „Musc A ster" genannt, hat einen Stamm ron 3,5-4 Meter (12 15) Höhe mit einem Durchmesser von. 0,5-0,6 Meter (2'). Ihr Holz ist prachtvoll geflammt, gut zu poliren und wird in Australien zur Fournirung von Pianos benutzt. Auch würde sich dasselbe sehr gut zu Bilderrahmen und zur Anfertigung von Holzpfeifen eignen.

Acacia homalophylla, Myall; von ihrem sehr schweren, schön gezeichneten und wohlriechenden Holze werden Tabakspfeifen verfertigt, sowie die $2^{\prime}$ langen Stiele der 12 15' langen Peitschen, welche von den Stationsbesitzern zum Eintreiben des wilden Viehes gebraucht werden. Gerade Stämme sind sehr seiten und erreichen nur eine Höhe von $10-12^{\prime}$ und kaum 1' Dicke. Diese Acacia wird hauptsächlich an den Flussgebieten des Murray gefunden und ist jetzt schon ein Ausfuhrartikel.

Santalum cognatum, das wohlriechende Santelholz, kommt ebenfalls hier ror, aber wie A cacia homalophylla nur in verkrüppelton Exemplaren. In Westaustralien hingegen wächst dieser Baum in grosser Anzahl und ziemlicher Grösse und ist schon seit langer Zeit ein Ausfuhrartikel.

Acacia pycnantha, 4-6 M. $\left(15-20^{\prime}\right)$ und Acacia mollissima W., $6-8$ M. $\left(20-30^{\prime}\right)$ hoch, liefern durch ihre Rinde ein ausgezeichnetes Gerbmittel, welches in grossen Massen in den Colonien verwendet wird. Oft findet man daher ganze Wälder von Acacien, welche ihrer Rinde beraubt worden und in Folge dessen abgestorben sind. Da jedoch die Leguminosen leicht aus Samen wachsen, so spriesst auch hier in kurzer Zeit wieder eine neue $\mathrm{Wal}$ dung empor.

A cacia verticillata W., den Bast der jungen, 6 8 M. $\left(20-30^{\prime}\right)$ hohen und $1 / 2{ }^{\prime}$ dicken Bäume fand Wil helmi in Gippsland ausserordentl. fest und könnte ders. wie Lindenbast zu Matten etc. verarbeitet werden.

Acacia dealbata Link, Silverwattle, wächst in Victoria am Yarra-Yarra-Flusse in einer Höhe von 8 M. $\left(30^{\prime}\right)$, einem Durchmesser des Stammes von $1-2^{\prime}$ und besitzt ein sehr festes Holz. 
Pittosporum bicolor Hook., Tolosatree und Exocarpus cupressiformis Labil., Sherrytree, erreichen eine Höhe von $5-8$ M. $\left(20-30^{\prime}\right)$ und eine Dicke des Stammes ron 0,28 M. Beide liefern ein zähes, helles Hol\%, welches sich sehr gut zu technischen Zwecken verwenden lässt.

Pittosporum undulatum Vent., Orangetree, sah Wilhelmi in den üppigen, feuchten Thälern von Gippsland 26 Meter $\left(80^{\prime}\right)$ hoch mit einer Dicke des Stammes von über 0,28 Meter, mit einer 17 Meter $\left(60^{\prime}\right)$ im Durchmesser haltenden Krone dicht mit weissen orangenartig riechenden Blüthen übersäet.

Prostanthera Lasianthus Labil., eine gewöhnlich nur als Strauch vorkommende Pflanze sah W. an dems. Orte als Baum von $20-26$ Meter $\left(70-80^{\prime}\right)$ und 0,28 Meter dick. Die aromatischen Blätter aller australischen Prostantheren würden ein schönes Parfüm liefern.

Pseudomoras australasica, der australische Maulbeerbaum, erreicht im Gebirge eine Höhe ron 12 15 Meter $\left(40-50^{4}\right)$ und 0,28 Meter Durchmesser. Das Holz ist unserem Lindenholze sehr ähnlich.

Unter den Coniferen ist noch zu bemerken:

Araucaria excelsa Ait., Norfolk Pine, welche auf $\mathrm{N}$ orfolk Is land eine Höhe von 70-85 Meter (250-300 Fuss) erreicht, mit einem Stamme von 1,3 Heter (4') Durchmesser.

Die herrliche Fächerpalme, Livistonia australis, erreicht eine Höhe von 14 Meter $\left(50^{\circ}\right)$ und liefert nicht allein Palmenkohl, sondern ans den Blättern derselben werden auch sehr danerhafte Hüte gefertigt.

H. $L$.

\section{Anthoxanthum odoratum L. (Ruchgras),}

bekannt wegen seines Gehaltes an Cumarin, wird nach Dr. Mehwald's Bericht von den Hausfrauen Norwegens $z$ wischen die Wäsche gelegt, um derselben guten Geruch zu ertheilen. (Isis, Dresden, Januar, Febr., März 1871.).

H. $L$. 